Bull. Chem. Soc. Ethiop. 2014, 28(3), 451-456.

Printed in Ethiopia

DOI: http://dx.doi.org/10.4314/bcse.v28i3.14

ISSN 1011-3924

(c) 2014 Chemical Society of Ethiopia

\title{
SYNTHESIS, CHARACTERIZATION AND ANTIBACTERIAL EVALUATION OF SOME NOVEL DERIVATIVES OF 2-BROMOMETHYL-BENZIMIDAZOLE
}

\author{
Abbas Ahmadi ${ }^{*}$ \\ Department of Medicinal Chemistry, Pharmaceutical Sciences Branch, Islamic Azad University,
} Tehran, Iran

(Received December 30, 2013; revised August 27, 2014)

\begin{abstract}
In the present study, synthesis, and biological evaluation of some novel derivatives of 2bromomethyl-benzimidazole were investigated. The structures of the new synthesized compounds were characterized by IR, ${ }^{1} \mathrm{H}-\mathrm{NMR},{ }^{13} \mathrm{C}-\mathrm{NMR}$, mass spectrometry and elemental analysis and screened for antimicrobial activity against two Gram-positive strains (Staphylococcus aureus and Bacillus subtilis) and two Gram-negative strains (Escherichia coli and Pseudomonas aeruginosa). Results indicate that new compounds showed moderate to good activity when compared with standard antibiotic Ciprofloxacin and Streptomycin.
\end{abstract}

KEY WORDS: Benzimidazole, Antibacterial activity, Bromo derivatives, Gram-positive strains, Gram-negative strains

\section{INTRODUCTION}

Infectious microbial diseases remain pressing problems worldwide, because resistance to a number of antimicrobial agents among variety of clinically significant species of microorganisms has become an important global health problem. One way to battle with this challenge is the conscious usage of the currently marketed antibiotics; the other is the development of novel antimicrobial agents [1]. The benzimidazole core is classified by medicinal chemists as one of the privileged sub-structures for drug design, in light of the affinity they display towards a variety of enzymes and protein receptors [2]. Benzimidazoles are heterocyclic compounds in which benzene is fused with five membered ring containing two heteroatoms. Both heteroatoms are nitrogen $(\mathrm{N})$, which are present at non-adjacent position [3]. The benzimidazole moiety is an important heterocyclic ring, a wide variety of benzimidazole derivatives are known for their chemotherapeutic importance and antimicrobial activities [4-9], antifungal activity [10-12], anti-inflammatory [13] and antioxidant [14-18] in this context. Due to great potential of the moiety, synthesis of benzimidazole derivatives was carried out to evaluate their antimicrobial. In generally benzimidazoles are readily formed by heating $o$ phenylenediamines with carboxylic acids or aldehydes. For example, benzimidazole itself is produced by heating $o$-phenylenedamine with $90 \%$ formic acid or formaldehyde [19].

In this research, some novel bromo-derivatives of 2-bromomethyl-benzimidazole (a-e in Figure 1) were synthesized and screened for antibacterial activity against various strains of Staphylococcus aureus, Bacillus subtilis, Escherichia coli and Pseudomonas aeruginosa.

\section{EXPERIMENTAL}

General. All chemicals and solvents were obtained from E-Merck and Sigma-Aldrich and used without further purification. All melting points are uncorrected and taken with an electrothermal melting point apparatus (Electrothermal Eng. Ltd, Essex, UK). IR spectra were determinate in $\mathrm{KBr}$ on a Shimadzu Dr-8031 instrument. The ${ }^{1} \mathrm{H}$ and ${ }^{13} \mathrm{C}-\mathrm{NMR}$ spectra of the synthesized compounds were measured in DMSO- $\mathrm{d}_{6}$ or $\mathrm{CDCl}_{3}$ solution and TMS as the internal standard using a Varian Mercury $400,400 \mathrm{MHz}$ instrument. All Chemical shifts were reported as $\delta$ (ppm)

*Corresponding author. E-mail: a-ahmadi@kiau.ac.ir; ahmadikiau@yahoo.com 
values. The mass spectra were recorded on a LCQ ion trap mass spectrometer (Thermo Fisher. San Jose.CA, USA), equipped with an EI source. Elemental analyses were carried out using a Perkin-Elmer, CHN elemental analyzer model 2400 and within $\pm 0.4 \%$ of the theoretical values.<smiles>OCc1nc2ccccc2[nH]1</smiles>

(I)<smiles>O=C(CBr)n1c(CBr)nc2ccccc21</smiles>

(a)

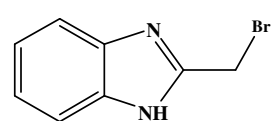

(II)

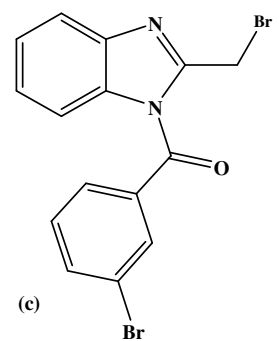

(b)

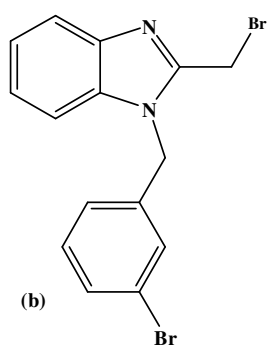

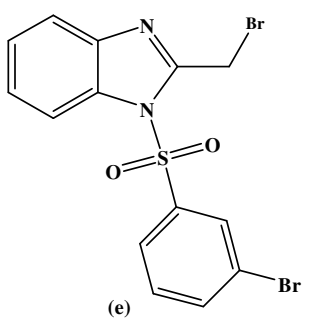

Figure 1. Structural formula for the intermediates (I and II) and final (a-e) synthesized compounds.

Synthesis of compounds $\boldsymbol{I}$ and $\boldsymbol{I I}$

(1H-Benzimidazol-2-yl)-methanol (I). This compound was prepared based on a known method with some modification $[19,20]$. Mixture of $o$-phenylenediamine $(27 \mathrm{~g}, 0.25 \mathrm{~mol})$ in $500 \mathrm{~mL} 4$ $\mathrm{M} \mathrm{HCl}$ and glycolic acid $(34.2 \mathrm{~g}, 0.45 \mathrm{~mol})$ in $250 \mathrm{~mL} 4 \mathrm{M} \mathrm{HCl}$ stirred and heated under reflux for $2 \mathrm{~h}$. Then slowly cooled and resultant mixture was basified with aqueous sodium hydroxide solution. The solid obtained, filtered, dried and re-crystallized with hot water (Scheme 1). (I: white to light brown powder, m.p. $171-174{ }^{\circ} \mathrm{C}, 89.1 \%$ yield).

2-Bromomethyl-1H-benzimidazole (II). Thionyl bromide is used for some brominations of certain $\alpha, \beta$-unsaturated carbonyls, and it also converts alcohols to alkyl bromides [21]. To a solution of I $(5.92 \mathrm{~g}, 0.04 \mathrm{~mol})$ in $75 \mathrm{~mL}$ cyclohexane as a nonpolar solvent and $2 \mathrm{~mL} \mathrm{N,N}$ dimethylformamide as a catalyst, was drop wise added thionyl bromide $(5 \mathrm{~mL})$. The addition of $\mathrm{N}, \mathrm{N}$-dimethylformamide as a catalyst was found to accelerate the reaction significantly through a Vilsmeier-Haack type $\mathrm{SOBr}_{2}-\mathrm{DMF}$ complex [22]. The mixture was refluxed for $10 \mathrm{~h}$. The excess of thionyl bromide was evaporated under vacuum and residue dissolved in cyclohexane and washed with water $(3 \times 50 \mathrm{~mL})$. The organic layer was dried over sodium sulfate and evaporated to obtain the product (Scheme 1). Beige to dark yellow powder, m.p. $158-162{ }^{\circ} \mathrm{C}$, 
89.1\% yield. IR (KBr, cm $\left.{ }^{-1}\right): 3345$ (N-H str), 3045 (Ar-C-H str), 2952 (aliphatic C-H str) , 1682 (C=N str), 1448 (C=C str), 1292 (C-N str), 667 (C-Br); ${ }^{1} \mathrm{H}-\mathrm{NMR}\left(\mathrm{CDCl}_{3}, \delta / \mathrm{ppm}\right): 4.64$ (s, 2H, $\left.\mathrm{CH}_{2}\right), 4.89(\mathrm{~s}, 1 \mathrm{H}, \mathrm{NH}), 7.20-7.25(\mathrm{~m}, 2 \mathrm{H}, \mathrm{Ar}-\mathrm{H}), 7.54-7.57(\mathrm{~m}, 2 \mathrm{H}, \mathrm{Ar}-\mathrm{H}) ;{ }^{13} \mathrm{C}-\mathrm{NMR}\left(\mathrm{CDCl}_{3}\right.$, $\delta / \mathrm{ppm}): 45.1\left(\mathrm{CH}_{2}-\mathrm{Br}\right), 114.8$ (benzimidazole, $\mathrm{CH}=$ ), 121.6 (benzimidazole, $\mathrm{CH}=$ ), 135.2 (benzimidazole, C), $140.1(\mathrm{~N}-\mathrm{C}=\mathrm{N})$. Anal. calcd. for $\mathrm{C}_{8} \mathrm{H}_{7} \mathrm{~N}_{2} \mathrm{Br}$ : C, 45.50; H, 3.31; N, $13.27 \%$. Found: C, 45.31; H, 3.28; N, 13.20\%. MS, $m / z: 211[\mathrm{M}]^{+}$.

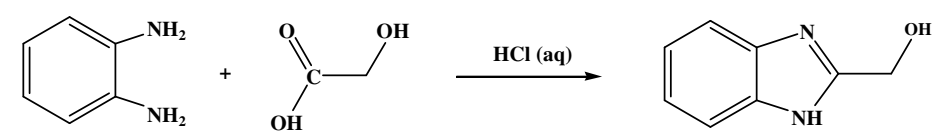

(I)

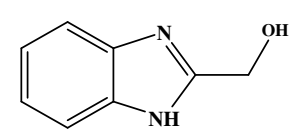

(I)

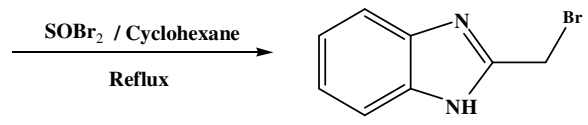

(II)

Scheme 1. Preparation for synthesis of intermediates (I and II).

General procedure for the preparation of the compounds (a-e). To a solution of II (10.5 g, 0.05 $\mathrm{mol})$, anhydrous potassium carbonate $(5.52 \mathrm{~g}, 0.04 \mathrm{~mol})$ in acetone $(25 \mathrm{~mL})$, appropriate $\mathrm{Br}-\mathrm{R}$ $\mathrm{Cl}(0.06 \mathrm{~mol})$ was added slowly (Scheme 2$)$. The mixture was refluxed for $12 \mathrm{~h}$ and hot mixture was poured into cold water $(300 \mathrm{~mL})$, extracted with ethyl acetate, dried over anhydrous sodium sulphate and concentrated under vacuum to give the desired product.

2-Bromo-1-(2-bromomethyl-benzimidazole-1-yl)-ethanone (a). Reddish yellow powder, m.p. 143-145 ${ }^{\circ} \mathrm{C}, 90.3 \%$ yield. IR $\left(\mathrm{KBr}, \mathrm{cm}^{-1}\right): 3078$ (Ar-C-H str), 2921 (aliphatic-C-H str), 1648(C=O), 1590 (C=N str), 1452 (C=C str), 1302 (C-N str), 675 (C-Br); ${ }^{1} \mathrm{H}-\mathrm{NMR}\left(\mathrm{CDCl}_{3}\right.$, $\delta / \mathrm{ppm}$ ): 4.61 (s, 2H, $\mathrm{CH}_{2}, \mathrm{Br}, \mathrm{C}=\mathrm{O}$ ), 4.75 (s, 2H, $\mathrm{CH}_{2}, \mathrm{Br}$ ), 7.21-7.28 (m, 2H, Ar-H), 7.60-7.70 (m, 2H, Ar-H); ${ }^{13} \mathrm{C}-\mathrm{NMR}\left(\mathrm{CDCl}_{3}, \delta / \mathrm{ppm}\right)$ : 29.4, $37.4\left(\mathrm{CH}_{2}-\mathrm{Br}\right), 119.8$ (benzimidazole, $\mathrm{CH}=$ ), 124.4 (benzimidazole, $\mathrm{CH}=$ ), 140.2 (benzimidazole, $\mathrm{C}), 145.8(\mathrm{~N}-\mathrm{C}=\mathrm{N}), 193.8(\mathrm{C}=\mathrm{O})$. Anal. calcd. for $\mathrm{C}_{10} \mathrm{H}_{8} \mathrm{~N}_{2} \mathrm{OBr}_{2}: \mathrm{C}, 36.15 ; \mathrm{H}, 2.40 ; \mathrm{N}, 8.43 \%$. Found: $\mathrm{C}, 36.05 ; \mathrm{H}, 2.28 ; \mathrm{N}, 8.35 \%$. MS, $\mathrm{m} / \mathrm{z}: 333[\mathrm{M}+\mathrm{H}]^{+}$.

1-(3-Bromobenzyl)-2-bromomethyl-1H-benzimidazole (b). Pale orange powder, m.p. 153-155 ${ }^{\circ} \mathrm{C}, 88.7 \%$ yield. IR $\left(\mathrm{KBr}, \mathrm{cm}^{-1}\right): 2966$ (Ar-C-H str), 2921 (aliphatic-C-H str), 1661(C=O), 1560 $\left(\mathrm{C}=\mathrm{N}\right.$ str), 1458 (C=C str), 1302 (C-N str), $675(\mathrm{C}-\mathrm{Br}) ;{ }^{1} \mathrm{H}-\mathrm{NMR}\left(\mathrm{CDCl}_{3}, \delta / \mathrm{ppm}\right): 4.56$ (s, 2H, $\left.\mathrm{CH}_{2}-\mathrm{Br}\right), 4.89\left(\mathrm{~s}, 2 \mathrm{H}, \mathrm{CH}_{2}-\mathrm{N}\right)$, 7.11-7.33 (m, 4H, Ar-H), 7.40-7.75 (m, 4H, benzimidazole); ${ }^{13} \mathrm{C}-$ NMR $\left(\mathrm{CDCl}_{3}, \delta / \mathrm{ppm}\right): 27.4\left(\mathrm{CH}_{2}-\mathrm{N}\right), 52.8\left(\mathrm{CH}_{2}-\mathrm{Br}\right), 119.8-133.1$ (phenyl, C), 123.8-138.3 (benzimidazole, C), $145.8(\mathrm{~N}-\mathrm{C}=\mathrm{N})$. Anal. calcd. for $\mathrm{C}_{15} \mathrm{H}_{12} \mathrm{~N}_{2} \mathrm{Br}_{2}: \mathrm{C}, 47.37 ; \mathrm{H}, 3.16 ; \mathrm{N}, 7.37 \%$. Found: C, 47.30; H, 3.02; N, 7.29\%. MS, $m / z: 381[\mathrm{M}+\mathrm{H}]^{+}$.

(2-Bromomethyl-benzimidazole-1-yl)-(3-bromophenyl)-methanone $\quad(\boldsymbol{c}) . \quad$ Yellowish orange powder, m.p. $160-162{ }^{\circ} \mathrm{C}, 87.3 \%$ yield. IR $\left(\mathrm{KBr}, \mathrm{cm}^{-1}\right)$ : 3077 (Ar-C-H str), 2965 (aliphatic-C-H str), $1720(\mathrm{C}=\mathrm{O}), 1580$ (C=N str), 1488 (C=C str), 1278 (C-N str), 690 (C-Br); ${ }^{1} \mathrm{H}-\mathrm{NMR}\left(\mathrm{CDCl}_{3}\right.$, $\delta / \mathrm{ppm}): 4.61$ (s, 2H, $\left.\mathrm{CH}_{2}-\mathrm{Br}\right), 7.40-7.75\left(\mathrm{~m}, 4 \mathrm{H}\right.$, benzimidazole), 7.30-7.95 (m, 4H, Ar-H); ${ }^{13} \mathrm{C}-$ NMR ( $\left.\mathrm{CDCl}_{3}, \delta / \mathrm{ppm}\right): 27.4\left(\mathrm{CH}_{2}-\mathrm{Br}\right), 114.8-136.1$ (benzimidazole, C),120.2-138.5 (phenyl, C), $143.1(\mathrm{~N}-\mathrm{C}=\mathrm{N}), 189.1(\mathrm{C}=\mathrm{O})$. Anal. calcd. for $\mathrm{C}_{15} \mathrm{H}_{10} \mathrm{~N}_{2} \mathrm{OBr}_{2}: \mathrm{C}, 45.68 ; \mathrm{H}, 2.53 ; \mathrm{N}, 7.10 \%$. Found: C, 45.60; H, 2.48; N, 7.05\%. MS, $m / z: 395[\mathrm{M}+\mathrm{H}]^{+}$. 
(5-Bromo-2-methoxy-phenyl)-(2-bromomethyl-benzimidazole-1-yl)-methanone $(\boldsymbol{d})$. Pale yellow powder, m.p. $155-160{ }^{\circ} \mathrm{C}, 85.9 \%$ yield. IR $\left(\mathrm{KBr}, \mathrm{cm}^{-1}\right)$ : 3067 (Ar-C-H str), 2945 (aliphatic-C-H str), 1730(C=O), 1562 (C=N str), 1490 (C=C str), 1201 (C-N str), 1118 (C-O str), 715 (C-Br); ${ }^{1} \mathrm{H}-\mathrm{NMR}\left(\mathrm{CDCl}_{3}, \delta / \mathrm{ppm}\right): 3.69\left(\mathrm{~s}, 3 \mathrm{H}, \mathrm{O}-\mathrm{CH}_{3}\right), 4.70\left(\mathrm{~s}, 2 \mathrm{H}, \mathrm{CH}_{2}-\mathrm{Br}\right), 6.85-7.90(\mathrm{~m}, 3 \mathrm{H}, \mathrm{Ar}-\mathrm{H})$, 7.25-7.80 (m, 4H, benzimidazole); ${ }^{13} \mathrm{C}-\mathrm{NMR}\left(\mathrm{CDCl}_{3}, \delta / \mathrm{ppm}\right): 26.9\left(\mathrm{CH}_{2}-\mathrm{Br}\right), 56.5(\mathrm{C}-\mathrm{O})$, 116.1-138.2 (benzimidazole, C), 113.9-139.4 (phenyl, C), $143.1(\mathrm{~N}-\mathrm{C}=\mathrm{N}), 164.6(\mathrm{Ph}-\mathrm{O}-), 192.2$ $(\mathrm{C}=\mathrm{O})$. Anal. calcd. for $\mathrm{C}_{16} \mathrm{H}_{12} \mathrm{~N}_{2} \mathrm{O}_{2} \mathrm{Br}_{2}: \mathrm{C}, 45.28 ; \mathrm{H}, 2.83 ; \mathrm{N}, 6.60 \%$. Found: C, 45.19; H, 2.79; $\mathrm{N}, 6.54 \%$. MS, $m / z: 425[\mathrm{M}+\mathrm{H}]^{+}$.

1-(3-Bromo-benzenesulfonyl)-2-bromomethyl-1H-benzenimidazole $(\boldsymbol{e})$. Cream white powder, m.p. $169-171{ }^{\circ} \mathrm{C}, 88.9 \%$ yield. IR $\left(\mathrm{KBr}, \mathrm{cm}^{-1}\right): 3319$ ( $\mathrm{S}=\mathrm{O}$ str), 3075 (Ar-C-H str), 2933 (aliphatic-C-H str), 1566 (C=N str), 1495 (C=C str), 1276 (C-N str), 690 (C-Br); ${ }^{1} \mathrm{H}-\mathrm{NMR}$ $\left(\mathrm{CDCl}_{3}, \delta / \mathrm{ppm}\right): 4.67\left(\mathrm{~s}, 2 \mathrm{H}, \mathrm{CH}_{2}-\mathrm{Br}\right), 7.21-7.80(\mathrm{~m}, 4 \mathrm{H}$, benzimidazole), 7.5-8.25 (m, 3H, $\mathrm{Ar}-\mathrm{H}) ;{ }^{13} \mathrm{C}-\mathrm{NMR}\left(\mathrm{CDCl}_{3}, \quad \delta / \mathrm{ppm}\right): 25.9\left(\mathrm{CH}_{2}-\mathrm{Br}\right), 122.9-142.4$ (Phenyl, C), 117.3-137.8 (benzimidazole, C), $141.8(\mathrm{~N}-\mathrm{C}=\mathrm{N})$. Anal. calcd. for $\mathrm{C}_{14} \mathrm{H}_{10} \mathrm{~N}_{2} \mathrm{O}_{2} \mathrm{SBr}_{2}: \mathrm{C}, 39.07 ; \mathrm{H}, 2.32 ; \mathrm{N}$, 6.51\%. Found: C, 38.95; H, 2.28; N, 6.48\%. MS, $m / z: 431[\mathrm{M}+\mathrm{H}]^{+}$.

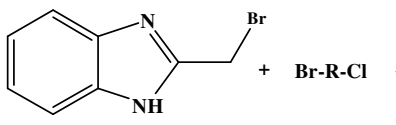

(II)

Br-R-Cl for (a-e) :

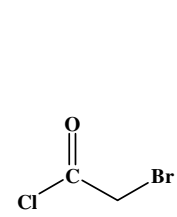

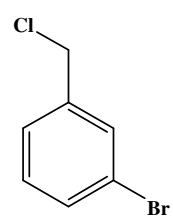

$\mathbf{b}$
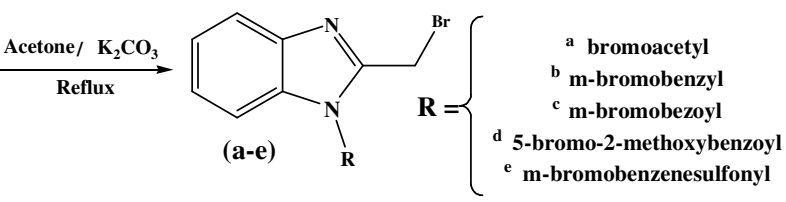

Scheme 2. Preparation rout for synthesis of new compounds (a-e).

Antimicrobial activity. The antibacterial activities of newly synthesized compounds were tested by paper disc diffusion method [23, 24]. Using nutrient agar medium against following microorganism: Staphylococcus aureus, Bacillus subtilis, (Gram positive) and Escherichia coli, Pseudomonas aeruginosa (Gram negative).

Experimental procedure. In the paper disc-diffusion method, paper disc impregnated with compounds dissolved in DMSO at conc. 50 and $100 \mu \mathrm{g} / \mathrm{mL}$ were used. The microorganism culture was spread over nutrient agar media in Petri dishes, and then the disc impregnated with the solution was placed on the surface of the media inoculated with the bacterial strain. The plates were incubated at $35{ }^{\circ} \mathrm{C}$ for $24 \mathrm{~h}$. After incubation, the zones of inhibition around the disc were observed. The zones of inhibition indicate that the compounds inhibit growth of microorganism. Each testing is done in triplicate. Streptomycin and Ciprofloxacin at conc. 50 and $100 \mu \mathrm{g} / \mathrm{mL}$ were used as standard drugs for antibacterial activity. Results were interpreted in terms of diameter $(\mathrm{mm})$ of zone of inhibition. 


\section{RESULTS AND DISCUSSIONS}

Chemistry. In continuation of our interest to explore of new antimicrobial drugs, the efficient syntheses of biologically active benzimidazole derivatives were applied in this study. Herein, some novel bromo-derivatives of 2-bromomethyl-benzimidazole (a-e) in good yields were prepared from 2-bromomethyl-1H-benzimidazole (II), appropriate bromo-hydrocarbon chloride and anhydrous potassium carbonate in acetone (Figure 2). Therefore, initially, $(1 \mathrm{H}-$ benzimidazol-2-yl)-methanol (I) was synthesized by coupling of $o$-phenylenediamine with organic acid based on a known method with some modification for increasing yield. TLC was used for monitoring the progress of the reaction; the structures of products were assessed by interpretation of obtained spectra. The spectroscopic data of IR, ${ }^{1} \mathrm{H}-\mathrm{NMR},{ }^{13} \mathrm{C}-\mathrm{NMR}$, mass spectra and elemental analysis were in agreement with the structure of the synthesized compounds.

Antimicrobial activities. For evaluation of the biological activities of the compounds, (a-e) were screened for in vitro antimicrobial activities against variety of bacteria, two Gram- positive strains (Staphylococcus aureus and Bacillus subtilis) and two Gram-negative strains (Escherichia coli and Pseudomonas aeruginosa). The preliminary screening results for the compounds (a-e) established that the newly synthesized compounds have not shown antibacterial activity against $E$. coli, but the compounds (a), (b) and (e) showed the best activities against $B$. Subtilis, and compounds (c) and (d) were slightly active against $B$. Subtilis. Compound (c) showed the best activity against $S$. Aureus, compounds (d) was slightly active against $S$. Aureus (Table 1 and 2).

Table 1. Zone of inhibition $(\mathrm{mm} \pm \mathrm{SD})$ of test sample and standard drug (Streptomycin and Ciprofloxacin) against $B$. subtilis and E. coli.

\begin{tabular}{|c|c|c|c|c|}
\hline \multirow{2}{*}{ Test samples } & \multicolumn{3}{|c|}{ Diameter of zone of inhibition in mm [mean \pm SD $(\mathrm{n}=3)]$} \\
\cline { 2 - 5 } & \multicolumn{2}{|c|}{ B. subtilis } & \multicolumn{2}{c|}{ E. coli } \\
\cline { 2 - 5 } & $50 \mu \mathrm{g} / \mathrm{mL}$ & $100 \mu \mathrm{g} / \mathrm{mL}$ & $50 \mu \mathrm{g} / \mathrm{mL}$ & $100 \mu \mathrm{g} / \mathrm{mL}$ \\
\hline I & $6.52 \pm 0.42$ & $10.281 \pm 0.42$ & $4.42 \pm 0.20$ & $8.60 \pm 0.42$ \\
\hline II & $6.90 \pm 1.30$ & $11.80 \pm 1.30$ & $5.15 \pm 0.50$ & $8.22 \pm 0.60$ \\
\hline $\mathbf{a}$ & $9.70 \pm 0.75$ & $13.95 \pm 1.20$ & $6.10 \pm 0.64$ & $9.25 \pm 0.45$ \\
\hline b & $9.75 \pm 0.44$ & $14.96 \pm 1.45$ & $4.40 \pm 1.25$ & $6.86 \pm 0.36$ \\
\hline c & $8.78 \pm 0.71$ & $12.84 \pm 1.30$ & $6.89 \pm 1.11$ & $11.10 \pm 0.46$ \\
\hline d & $8.32 \pm 0.36$ & $12.88 \pm 0.32$ & $6.88 \pm 0.78$ & $11.66 \pm 0.35$ \\
\hline e & $9.85 \pm 0.66$ & $15.10 \pm 0.68$ & $3.76 \pm 0.38$ & $8.20 \pm 0.45$ \\
\hline Ciprofloxacin & $14.36 \pm 0.36$ & $19.24 \pm 0.35$ & $11.36 \pm 0.44$ & $16.45 \pm 0.28$ \\
\hline Streptomycin & $13.55 \pm 0.44$ & $18.57 \pm 0.52$ & $10.80 \pm 0.54$ & $15.85 \pm 0.35$ \\
\hline
\end{tabular}

Table 2. Zone of inhibition $(\mathrm{mm} \pm \mathrm{SD})$ of test sample and standard drug (Streptomycin and Ciprofloxacin) against $S$. Aureus and $P$. aeureginosa.

\begin{tabular}{|c|c|c|c|c|}
\hline \multirow{2}{*}{ Test samples } & \multicolumn{3}{|c|}{ Diameter of zone of inhibition in mm [mean \pm SD $(\mathrm{n}=3)]$} \\
\cline { 2 - 5 } & \multicolumn{2}{|c|}{ S. Aureus } & \multicolumn{2}{c|}{ P. aeureginosa } \\
\cline { 2 - 5 } & $50 \mu \mathrm{g} / \mathrm{mL}$ & $100 \mu \mathrm{g} / \mathrm{mL}$ & $50 \mu \mathrm{g} / \mathrm{mL}$ & $100 \mu \mathrm{g} / \mathrm{mL}$ \\
\hline I & $6.54 \pm 0.58$ & $11.26 \pm 0.23$ & $4.75 \pm 0.63$ & $7.53 \pm 0.54$ \\
\hline a & $7.70 \pm 0.42$ & $14.70 \pm 1.00$ & $8.00 \pm 0.36$ & $12.92 \pm 0.78$ \\
\hline b & $6.80 \pm 0.55$ & $13.14 \pm 0.33$ & $8.85 \pm 0.85$ & $14.80 \pm 1.00$ \\
\hline c & $5.34 \pm 0.78$ & $11.85 \pm 1.20$ & $6.80 \pm 0.62$ & $12.78 \pm 1.25$ \\
\hline d & $8.62 \pm 0.44$ & $14.45 \pm 0.35$ & $8.78 \pm 0.71$ & $14.95 \pm 1.15$ \\
\hline e & $7.85 \pm 0.80$ & $12.76 \pm 0.25$ & $6.24 \pm 0.26$ & $12.95 \pm 0.29$ \\
\hline Ciprofloxacin & $5.22 \pm 1.20$ & $10.24 \pm 0.76$ & $4.00 \pm 0.30$ & $7.86 \pm 0.34$ \\
\hline Streptomycin & $11.95 \pm 0.22$ & $17.45 \pm 0.22$ & $14.00 \pm 0.28$ & $19.12 \pm 0.32$ \\
\hline
\end{tabular}

Bull. Chem. Soc. Ethiop. 2014, 28(3) 


\section{CONCLUSIONS}

A series of some novel bromo-derivatives of 2-bromomethyl-benzimidazole were synthesized and evaluated for their potential antimicrobial activities. Based on results, it can be concluded that all the synthesized compounds showed good to moderate antimicrobial activities. The results indicated that new antimicrobial compounds could be prepared by changing of different substrates on various benzimidazole derivatives. These new compounds could be evaluated for further pharmacological activities in other studies.

\section{ACKNOWLEDGEMENT}

The author gratefully acknowledges beneficial support of Pharmaceutical Sciences Branch, Islamic Azad University for this research project.

\section{REFERENCES}

1. Ozkay, Y.; Tunali, Y.; Karaca, H.; Srikdag, I. Eur. J. Med. Chem. 2010, 45, 3293.

2. Varala, R.; Enugala, R. Chem. Pharm. Bull. 2007, 55, 1254.

3. Dubey, R.; Moorthy N.S.H.N. Chem. Pharm. Bull. 2007, 55, 115.

4. Utku, S.; Gokce, M.; Ozcelik, B.; Bercin, E. Turk. J. Pharm. Sci. 2008, 5, 107.

5. Nakano, H.; Inoue, T.; Kawasaki, N.; Miyataka, H.; Matsumoto, H.; Taguchi, T.; Inagaki, N.; Nagai, H.; Satoh, T. Chem. Pharm. Bull. 1999, 47, 1573.

6. Habernickel, V.J. Drugs Made in Germany 1992, 35, 97.

7. Islam, I.; Skibo, E.B.; Dorr, R.T.; Alberts, D.S. J. Med. Chem. 1991, 34, 2954.

8. Kruse, L.L.; Ladd, D.L.; Harrsch, R.B.; McCabe, F.L.; Mong, S.M.; Faucette, L. J. Med. Chem. 1989, 32, 409.

9. Fukuda, T.; Morimoto, Y.; Iemura, R.; Kawashima, T.; Tsukamoto, G.; Ito, K. Drug Res. 1984, 34, 801.

10. Ayhan-Kilcigil, G.; Kus, C.; Çoban, T.; Can-Eke, B. J. Enzym. Inhib. Med. Ch. 2004, 19, 129.

11. Kus, C.; Ayhan-Kilcigil, G.; Can-Eke, B.; Iscan, M. Arch. Pharm. Res. 2004, 27, 156.

12. Can-Eke, B.; Puskullu, M.O.; Buyukbingol, E.; Iscan, M. Chem-Bio Interact. 1998, 113, 65.

13. Göker, H.; Kilcigil, G.A.; Tuncbilek, M.; Kus, C.; Ertan, R.; Kendi, E.; Özbey, S.; Fort, M.; Garcia, C.; Farré, A.J. Heterocycles 1999, 51, 2561.

14. Göker, H.; Kus, C.; Boykin, D.W.; Yildiz, S.; Altanlar, N. Biorg. Med. Chem. 2002, 10, 2589.

15. Habib, N.S.; Abdel-Hamid, S.; El-Hawash, M. Farmaco, 1989, 44, 1225.

16. Coburn, R.A.; Clark, M.T.; Evans, R.T.; Genco, R.J. J. Med. Chem. 1987, 30, 205.

17. Soliman, F.S.G.; Rida, S.M.; Badawey, E.A.M.; Kappe, T. Arch. Pharm. 1984, 317, 951.

18. Abdel-Rahman, A.E.; Mahmoud, A.M.; El-Naggar, G.M.; El-Sherief, H.A. Pharmazie 1983, 38, 589.

19. Finar, I.L. Organic Chemistry: Stereochemistry and the Chemistry of Natural Products, Vol. 2, 5th ed., John Wiley and Sons: New York; 1975; p 631.

20. Furniss, B.S.; Hannaford, A.J.; Smith, P.W.G.; Tatchell, A.R. Vogel's Textbook of Practical Organic Chemistry, Vol. 5, Longman-ELBS: London; 2007; p 1162.

21. Mundy, B.P. Thionyl Bromide in Encyclopedia of Reagents for Organic Synthesis, Paquette, E. (Ed.), John Wiley and Sons: New York; 2004.

22. Advait, S.N.; Ralph, N.S.; Byong-Don, Ch.; Kyung, W.J. Tetrahedron. Lett. 2000, 41, 3011.

23. Pharmacopoeia of India, Vol II, Govt. of India, Ministry of Health and Family Welfare: New Delhi; 1996; p A-111.

24. Srivastava, S.K.; Verma, S.; Srivastava, S.D. J. Chem. Pharm. Res. 2010, 2, 270. 